TITLE:

\title{
Non-inductive variable reactor design and computer simulation of rectifier type superconducting fault current limiter
}

\section{AUTHOR(S):}

Hoshino, T; Muta, I; Nakamura, T; Salim, KM; Yamada, $M$

\section{CITATION:}

Hoshino, T ...[et al]. Non-inductive variable reactor design and computer simulation of rectifier type superconducting fault current limiter. IEEE TRANSACTIONS ON APPLIED SUPERCONDUCTIVITY 2005, 15(2): 2063-2066

\section{ISSUE DATE:}

2005-06

URL:

http://hdl.handle.net/2433/39961

\section{RIGHT:}

(c)2005 IEEE. Personal use of this material is permitted. However, permission to reprint/republish this material for advertising or promotional purposes or for creating new collective works for resale or redistribution to servers or lists, or to reuse any copyrighted component of this work in other works must be obtained from the IEEE. 


\title{
Non-Inductive Variable Reactor Design and Computer Simulation of Rectifier Type Superconducting Fault Current Limiter
}

\author{
Tsutomu Hoshino, Itsuya Muta, Member, IEEE, Taketsune Nakamura, Khosru M. Salim, and Masato Yamada
}

\begin{abstract}
A rectifier type superconducting fault current limiter with noninductive reactor has been proposed by the authors. The concept behind this SFCL is that the high impedance generated during superconducting to normal state of the trigger coil limits the fault current. In the hybrid bridge circuit of the SFCL, two superconducting coils: a trigger coil and a limiting coil are connected in anti-parallel. Both the coils are magnetically coupled with each other and could have the same value of self inductance so that they can share the line current equally. At fault time when the trigger coil current reaches a certain level, the trigger coil changes from superconducting state to normal state. This super to normal transition of the trigger coil changes the current ratio of the coils and therefore the flux inside the reactor is no longer zero. So, the equivalent impedance of both the coils is increased and limits the fault current. We have carried out computer simulation using PSCAD/EMTDC and observed the results. Both the simulation and preliminary experiment shows good results. The advantage of using hybrid bridge circuit is that the SFCL can also be used as circuit breaker.
\end{abstract}

Index Terms-Bridge rectifier, fault current limiter, noninductive reactor, superconductor, super-to-normal transition, trigger coil.

\section{INTRODUCTION}

$\mathbf{M}$ ANY circuits and configurations for superconducting fault current limiter (SFCL) have been proposed and studied today, some known as rectifier types [1], [2]. The rectifier type SFCL is compatible with the semiconductor switchgear, and free from the quench phenomenon. Experimental tests with a large inductance coil revealed that unwanted limiting operation occurred with abrupt increases of current-demand [3]. One solution to avoid this problem is applying variable reactor to the DC coil, which is the key element of rectifier type SFCL. To implement variable reactor, some configurations are proposed [4]-[6], tested [7]-[9], and designed [10]. This reactor has low inductance when normal operation (wintind mode) and high inductance when the current through

Manuscript received October 5, 2004. This work was supported in part by Kansai Electric Power Co. and in part by 21st Century COE under Program 14213201.

T. Hoshino and T. Nakamura are with the Graduate School of Engineering, Kyoto University, Kyoto 615-8510, Japan (e-mail: hoshino@kuee.kyoto-u. ac.jp; tk_naka@kuee.kyoto-u.ac.jp).

I. Muta is with Saga University, Saga 840-8502, Japan (e-mail: muta128@bb.excite.co.jp).

K. M. Salim is with School of Communication, Independent University, Baridhara, Dhaka 1212, Bangladesh (e-mail: khosru@iub.edu.bd).

M. Yamada is with General R\&D Center, Kansai Electric Power Co., Amagasaki, Hyogo 661-0974, Japan (e-mail: K431639@kepco.co.jp).

Digital Object Identifier 10.1109/TASC.2005.849452

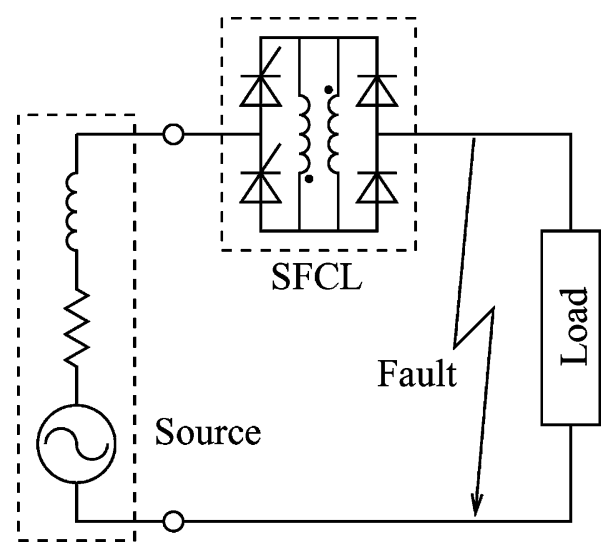

Fig. 1. Basic circuit diagram of noninductive DC rectifier type SFCL in a single phase circuit, where the source are indicated as an ideal voltage source and source impedance.

the reactor exceed certain value due to the fault. Low inductance value of the reactor allow to follow the current increase due to poor current limiting performance. In the case of the fault, the increased inductance of the reactor brings out current limiting faculty.

Non-inductive reactor is made of two superconducting coils. A trigger coil and a limiting coil are connected in anti-parallel and are magnetically coupled well. It is used for variable reactor as shown in Fig. 1. The power source is indicated as an ideal voltage source with a source impedance. The fault point has set at most severe position that is just the load-side terminal. In the normal mode operation, both coils are in superconducting state and noninductive reactor shows its leakage inductance, which is relatively small. In the limiting mode, the trigger coil becomes normal-conducting state due to the current through the coil reaching its critical current. The self-inductance of the limiting coil acts as a reactor as the common rectifier type SFCL. A small-scale experiment was performed using the superconducting transformer with four wound windings, where a single hybrid bridge circuit was used for both the coils [9]. The experimental results confirmed that the SFCL works in current limiting mode after quenches of the trigger coil. It was also confirmed that the SFCL can work as a half-cycle circuit breaker as well as current-limiter. In the fault test, the current limiting ratio was poor because the trigger coil used in the experiment had very high critical current capacity. The current limiting ratio could be adjusted by the proper selection (the critical current) of the trigger coil. 


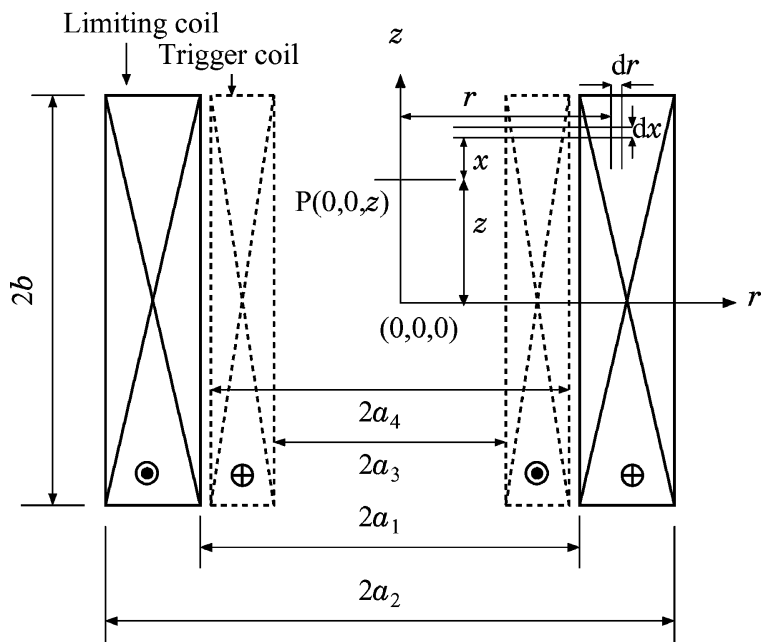

Fig. 2. Coil design 1: two coils are separately fabricated and placed them co-axially so that their magnetic field would oppose each other.

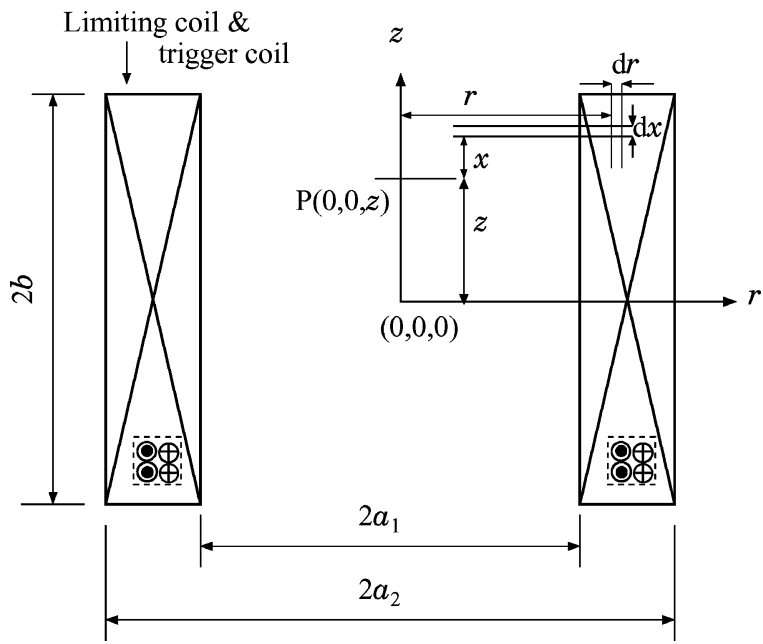

Fig. 3. Coil design 2: two coils are fabricated as single unit. The sum of anti-parallel coils current is zero.

In this paper, coil design for $6.6 \mathrm{kV} / 1 \mathrm{kA}$ rated noninductive reactor and the analytical results using computer software PSCAD/EMTDC are described.

\section{COIL DEsign fOR A $6.6 \mathrm{kV} / 1 \mathrm{kA}$ RATED NON-INDUCTIVE REACTOR}

The coils of the noninductive reactor share the line current equally during normal operational time. The self inductance of each coil should be equal (to avoid the circulating current). Compact design is necessary, to reduce the leakage inductance, as well as the volume of the cryogenic system. However, the maximum flux density should not cross a limit during fault time that might quench the limiting coil. In case of $\mathrm{Nb}-\mathrm{Ti}$, the maximum flux density should not exceed a value of $8 \mathrm{~T}$. For HTS bulk material, this value is lower at higher temperature. The inductor could be manufactured in two ways. The main and trigger coil are separately fabricated and could be placed coaxially as shown in Fig. 2. The other way, both the coils could be constructed as a single unit, which is a bifilar winding, as shown in the Fig. 3. The first one is easier to construct, and the temperature rise in the trigger coil does not affect the limiting coil during fault time. However the leakage inductance would be higher (because of the gap between the two coils). The latter could be made with higher coupling factor, generating less leakage inductance.

The flux density $B_{z}(z)$ at point $\mathrm{P}(0,0, z)$ along the $z$ axis produced with the limiting coil as shown in Fig. 2 is expressed as follows,

$$
B_{z}(z)=\mu_{0} \int_{a_{1}}^{a_{2}} \mathrm{~d} r \int_{-b-z}^{b-z} \frac{N r^{2} J}{4 b\left(a_{2}-a_{1}\right)\left(r^{2}+x^{2}\right)^{\frac{3}{2}}} \mathrm{~d} x
$$

where $2 b$ is the height of the coil, $a_{1}$ is inner diameter of the limiting coil, $a_{2}$ is outer diameter of the limiting coil, $N$ is number of turns, $(r, x+z)$ is coil fragment position, $J$ is the current density at $(r, x+z)$.

Considering overall current density ' $\lambda j$ ' where $\lambda$ is the space factor and $\alpha=a_{2} / a_{1}, \beta=b / a_{1}$ and $Z=z / a_{1}$ the flux density at the point $\mathrm{P}$ is as follows,

$$
\begin{aligned}
& B_{z}(z)=\mu_{0} \frac{a_{1} \lambda j}{2}\left\{(Z+\beta) \ln \frac{\alpha+\sqrt{\alpha^{2}+(Z+\beta)^{2}}}{1+\sqrt{1+(Z+\beta)^{2}}}\right. \\
&\left.-(Z+\beta) \ln \frac{\alpha+\sqrt{\alpha^{2}+(Z-\beta)^{2}}}{1+\sqrt{1+(Z-\beta)^{2}}}\right\} .
\end{aligned}
$$

Flux density at the center of the coil $(z=0)$ is obtained from (2) as follows,

$$
B_{z}(0)=\mu_{0} a_{1} \lambda j \beta \ln \frac{\alpha+\sqrt{\alpha^{2}+\beta^{2}}}{1+\sqrt{1+\beta^{2}}} .
$$

The self and leakage inductance of each coil are required to calculate. The flux linkage $\phi_{11}$ of the limiting coil can be express by the following equation;

$$
\phi_{11}=\int_{-b}^{b} \mathrm{~d} z \int_{0}^{a_{2}} B_{z}(r, x) f_{l}(r) 2 \pi r \mathrm{~d} r .
$$

Similarly for the flux linkage $\phi_{22}$ of the trigger coil is

$$
\phi_{22}=\int_{-b}^{b} \mathrm{~d} z \int_{0}^{a_{4}} B_{z}(r, x) f_{t}(r) 2 \pi r \mathrm{~d} r
$$

where $f_{l}(r)$ is the number of turns in per unit length of the limiting coil that can be expressed as

$$
f_{l}(r)= \begin{cases}\frac{N_{l}}{2 b} & \text { if } 0 \leq r<a_{1} \\ \frac{N_{l}\left(a_{2}-r\right)}{b\left(r-a_{1}\right)} & \text { if } a_{1} \leq r \leq a_{2} \\ 0 & \text { if } a_{2}<r\end{cases}
$$

where $N_{l}$ is turn number of limiting coil. Similarly for trigger coil $f_{t}(r)$ can be written as

$$
f_{t}(r)= \begin{cases}\frac{N_{t}}{2 b} & \text { if } 0 \leq r<a_{3} \\ \frac{N_{t}\left(a_{2}-r\right)}{b\left(r-a_{1}\right)} & \text { if } a_{3} \leq r \leq a_{4} \\ 0 & \text { if } a_{4}<r\end{cases}
$$

where $N_{t}$ is turn number of trigger coil. 
TABLE I

Designed Single CoIl PARAMETERs, Where ITS CURRENT is $1 \mathrm{kA}$

\begin{tabular}{ccccccc}
\hline $\begin{array}{c}r_{d} \\
\mathrm{~mm}\end{array}$ & $\begin{array}{c}d_{d} \\
\mathrm{~mm}\end{array}$ & $\begin{array}{c}2 b \\
\mathrm{~mm}\end{array}$ & $N$ & $\begin{array}{c}L_{d} \\
\mathrm{mH}\end{array}$ & $\begin{array}{c}B_{0} \\
\mathrm{~T}\end{array}$ & $\begin{array}{c}J_{e} \\
\mathrm{~A} / \mathrm{mm}\end{array}$ \\
\hline 195 & 10 & 100 & 300 & 48.0 & 0.937 & 300 \\
190 & 20 & 100 & 310 & 47.3 & 0.992 & 155 \\
100 & 20 & 150 & 210 & 6.54 & 1.06 & 70 \\
140 & 20 & 150 & 300 & 23.2 & 1.19 & 100 \\
130 & 20 & 140 & 280 & 18.6 & 1.19 & 100 \\
130 & 25 & 200 & 240 & 11.0 & 0.92 & 48 \\
140 & 15 & 200 & 300 & 20.1 & 1.10 & 100 \\
150 & 20 & 224 & 360 & 29.9 & 1.21 & 80.4 \\
160 & 30 & 200 & 240 & 15.2 & 0.8 & 40 \\
180 & 20 & 180 & 224 & 17.5 & 0.7 & 62.2 \\
175 & 40 & 50 & 200 & 15.9 & 0.71 & 100 \\
250 & 60 & 350 & 200 & 15.1 & 0.41 & 9.52 \\
280 & 80 & 350 & 200 & 17.6 & 0.38 & 7.14 \\
\hline
\end{tabular}

The flux linkage $\phi_{12}$ of the limiting coil induced by the trigger coil can be expressed by the following equation,

$$
\phi_{12}=\int_{-b}^{b} \mathrm{~d} z \int_{0}^{a_{4}} B_{z}(r, x) f_{t}(r) 2 \pi r \mathrm{~d} r
$$

Similarly

$$
\phi_{21}=\int_{-b}^{b} \mathrm{~d} z \int_{0}^{a_{2}} B_{z}(r, x) f_{l}(r) 2 \pi r \mathrm{~d} r
$$

Assuming the limiting and trigger coil current are equal to unity, the self inductance of the limiting coil $\left(L_{1}\right)$ and trigger coil $\left(L_{2}\right)$ and their mutual inductance $(M)$ can be calculated by the following equations. $L_{1}=\phi_{11}, L_{2}=\phi_{22}$ and $M=\sqrt{M_{12} M_{21}}=$ $\sqrt{\phi_{12} \phi_{21}}$. As the magnetic field of each coil is anti-parallel, the conditions to get zero field at the center point can be calculated.

The impedance of this SFCL is not zero in the normal operational condition because there are always some magnetic flux in the space in between the two coils, which caused the leakage inductance. If the coils are fabricated as a single unit (bifilar winding) as shown in Fig. 3, a lower leakage inductance can be achieved, but has difficulty of the insulation between two coils. In such a case, the same thickness of the winding are shared by the two coils. Equal turns per unit cross section of the windings is necessary for main and trigger coil to produce equal inductance. At normal condition, the coil current $i=i_{1}-i_{2}=0$ so the (3) gives zero flux density for the design shown in Fig. 3. At fault time, after super-to-normal transition of the trigger coil $\left(i_{2}=0\right)$, the flux density along $z$ axis increases with the increase of current though the main coil. Based on the above equations, the possible design parameter sets of a winding are calculated in Table I for a $6.6 \mathrm{kV}, 1 \mathrm{kA}$ class SFCL considering $i_{1}=1 \mathrm{kA}$ and $i_{2}=0$. For $\mathrm{Nb}-\mathrm{Ti}$, the flux density of $1.0 \mathrm{~T}$ or less is a reasonable value at the center point of the coil, where $r_{d}$ is the average radius such as $\left(a_{1}+a_{2}\right) / 2$ or $\left(a_{3}+a_{4}\right) / 2, d_{d}$ is the thickness of the coil such as $a_{2}-a_{1}$ or $a_{4}-a_{3}, L_{d}$ is self inductance of the coil, $J_{e}$ is over all current density. If the limited fault current goes up to 3 or 4 times of the rated $1 \mathrm{kA}$, the flux density also increases 3 to 4 times but still remain below its critical value around $8 \mathrm{~T}$. The last two parameter sets of the

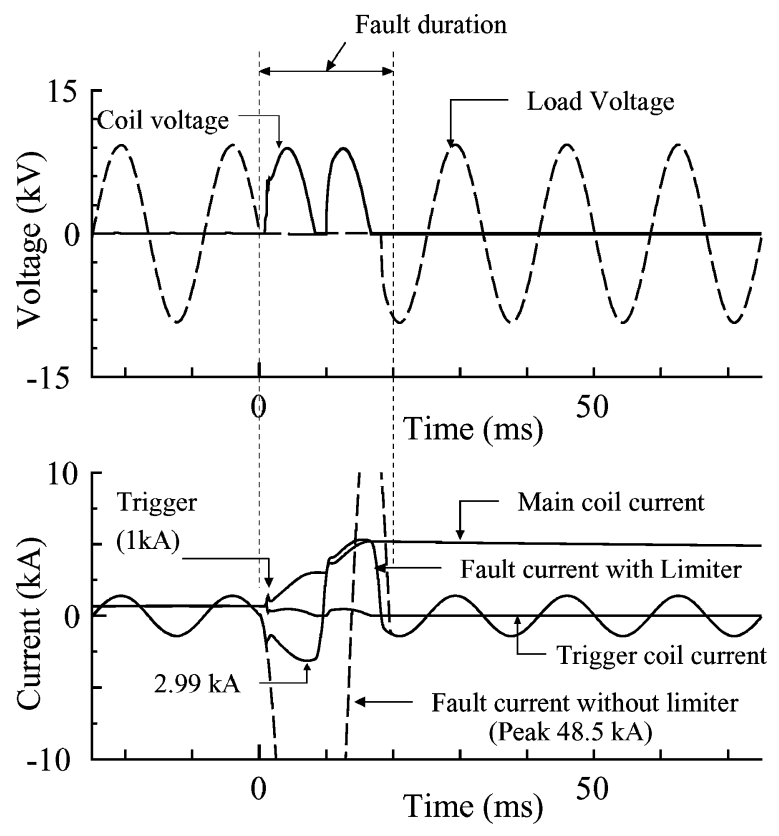

Fig. 4. Simulation result of a $6.6 \mathrm{kV}, 1 \mathrm{kA}$ system with zero crossing fault.

Table I represent feasible designs using HTS superconducting tape where low electric field intensity is required.

\section{PSCAD/EMTDC ANALYSIS RESULTS OF A 6.6 kV, 1 kA CLASS SFCL}

Non-inductive reactor shows only the leakage inductance during normal operation. The self inductance of the limiting coil is the maximum inductance that could be seen during current limiting mode at fault condition with the test circuit as shown in Fig. 1. We performed simulations, using EMTDC (Electro-Magnetic Transient for DC system) for a $6.6 \mathrm{kV}, 1 \mathrm{kA}$ system, with 5 percent source inductance. The inductance of each coil is assumed to be $17.5 \mathrm{mH}(=1 \mathrm{pu})$, with a coupling factor of 0.9. The critical current of the trigger coil was set to $1.0 \mathrm{kA}$. Fig. 4 illustrates the output waveforms of the simulation. A short term (1 cycle) zero crossing fault was made. The voltage waveforms are presented in the upper curves, with current waveforms in the lower curves. Before the fault, both coil current waveforms stay on $0.7 \mathrm{kA}$. The waveforms were slightly rippled. In the first half-cycle of the fault, the peak current reached only $2.99 \mathrm{kA}$ with SFCL, while it was $48.5 \mathrm{kA}$ without the SFCL. The average current increase rate was $2 \mathrm{kA} /$ half-cycle with 1 pu limiting inductance. When the load current reached $40 \%$ over its rated peak value, the SFCL entered limiting mode. And after one cycle, the peak current reached 3.6 times larger than rated peak value. After release of the fault, the main coil current flows through the diode leg of the bridge. The current decays slowly. In Fig. 5, the waveforms are obtained when fault is made at $90^{\circ}$ of the current phase. This time, the fault duration is set for 1.25 cycle. The limited fault current has reached to $2.0 \mathrm{kA}$ in the first half-cycle where this value is $44.5 \mathrm{kA}$ without SFCL. The reason of $1 / 4$ cycle delay to shutdown the fault is the delay of turn off the thyristor which simulate the short-circuited fault. Increasing the value of limiting inductance up to $4 \mathrm{pu}$, the fault current was less than 


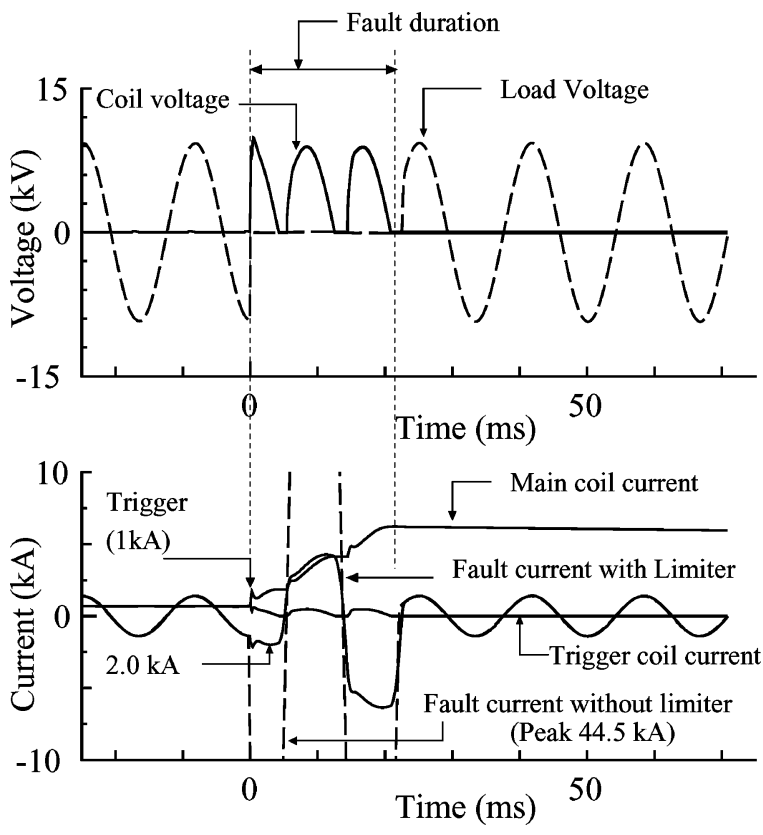

Fig. 5. Simulation result of a $6.6 \mathrm{kV}, 1 \mathrm{kA}$ system with $90^{\circ}$ fault.

$2 \mathrm{kA}$. A limiting inductance value of $1 \mathrm{pu}$, is a moderate value, with smaller cold mass for the system.

\section{CONCLUSION}

Basic coil design for $6.6 \mathrm{kV} / 1 \mathrm{kA}$ rated noninductive reactor has been carried out. Two configuration of the noninductive reactor for rectifier type SFCL has been proposed. Coaxial coil arrangement and bifilar winding arrangement were compared. Bifilar winding arrangement was superior to have high impedance ratio (normal operating mode and current limiting mode), but coaxial arrangement is selected in the point of the insulation. The total loss in the cryogenic environment should be accumulated to design cooling system. The PSCAD/EMTDC simulations have been carried out with the designed coil and the current limiting performances have been evaluated. With five percent source inductance, adequate inductance value $1 \mathrm{pu}$ $(=17.5 \mathrm{mH})$ was obtained.

The insulation layer was considered as a lower current density of the winding. Design of the insulator and electric field analysis are expected to be performed in consecutive work.

\section{REFERENCES}

[1] H. J. Boening and D. A. Paice, "Fault current limiter using superconducting coil," IEEE Trans. Magn., vol. MAG-19, no. 3, pp. 1051-1053, May 1983.

[2] T. Yazawa and E. Yoneda et al., "Design and test results of $6.6 \mathrm{kV}$ high-Tc superconducting fault current limiter," IEEE Trans. Appl. Supercond., vol. ASC-11, no. 1, pp. 2511-2514, Mar. 2001.

[3] T. Hoshino, K. M. Salim, M. Nishikawa, I. Muta, and T. Nakamura, "DC reactor effect on bridge type superconducting fault current limiter during load increasing," IEEE Trans. Appl. Superconduct., vol. ASC-11, no. 1, pp. 1944-1947 (2001.3).

[4] - "Proposal of saturated DC reactor type superconducting fault current limiter (SFCL)," Cryogenics, vol. 41, no. 7, pp. 469-474, Jul. 2001.

[5] I. Muta, T. Hoshino, K. M. Salim, A. Kawasaki, and T. Nakamura, "Proposal of DC shield reactor type superconducting fault current limiter," Cryogenics, vol. 44, no. 3, pp. 177-182, Mar. 2004.

[6] K. M. Salim, I. Muta, T. Hoshino, A. Kawasaki, and T. Nakamura, "Proposal of rectifier type fault current limiter with noninductive reactor (SFCL)," Cryogenics, vol. 44, no. 3, pp. 171-176, Mar. 2004.

[7] K. M. Salim, T. Hoshino, M. Nishikawa, I. Muta, and T. Nakamura, "Preliminary experiments on saturated DC reactor type fault current limiter," IEEE Trans. Appl. Supercond., vol. ASC-12, no. 1, pp. 872-875, Mar. 2002.

[8] T. Hoshino, K. M. Salim, I. Muta, and T. Nakamura, "Experiment using variable reactor of rectifier type superconducting fault current limiter with a short-circuited trigger coil," IEEE Trans. Appl. Supercond., vol. ASC-14, no. 2, pp. 626-629, Jun. 2004.

[9] K. M. Salim, T. Hoshino, I. Muta, T. Nakamura, and M. Yamada, "Low voltage test of a single bridge rectifier type superconducting fault current limiter with noninductive reactor," in 6th Eur. Conf. Applied Superconductivity, [4-9], Sep. 18, 2003.

[10] T. Hoshino, A. Kawasaki, M. Nishikawa, K. M. Salim, I. Muta, and T. Nakamura, "Design of $6.6 \mathrm{kV}, 100 \mathrm{~A}$ saturated DC reactor type superconducting fault current limiter," IEEE Trans. Appl. Supercond., vol. ASC-13, no. 2, pp. 2012-2015, Jun. 2003. 